Ann Zootech, I979, 28 (4), 459-453.

Note

\title{
Evacuation gastrique après déafférentation vagale subtotale chez le Porc
}

\author{
J.P. LAPLACE, J.C. CUBER et P.A. VILLIERS \\ Laboratoive de Physiologie de la Nutrition, \\ Centre national de Recherches zootechniques, I.N.R.A., \\ $7835^{\circ}$ Jouy-en-Josas (France)
}

\begin{abstract}
Résumé
L'évacuation gastrique de la matière sèche est étudiée chez 3 porcs équipés d'une canule gastrique permanente et soumis à déafférentation vagale subtotale. $\mathrm{I}_{A}$ a suppression chirurgicale de la majeure partie des afférences vagales entraîne un important ralentissement de l'évacuation gastrique. L'apparente opposition entre l'hyperactivité antrale mise en évidence antérieurement et ce ralentissement de l'évacuation gastrique est discutée en relation avec le rôle des décharges vagales efférentes dans l'organisation de la motricité d'une part, et avec le rôle des afférences provenant de l'estomac et du duodénum dans la modulation de ces décharges efférentes.
\end{abstract}

\section{Introduction}

Une technique de déafférentation vagale des organes postdiaphragmatiques du Porc a été antérieurement proposée (LAPLACE et DARCY, I978; DARCY et al., I979). Elle permet de supprimer sélectivement les afférences vagales d'origine digestive (intéroception empruntant la voie du nerf pneumogastrique) en préservant à la fois l'innervation vagale du bloc cardiopulmonaire et une partie des efférences vagales (motrices) destinées aux organes post diaphragmatiques. Cette déafférentation (DARCy et al., I979) est dite subtotale en raison de l'existence de rameaux de communication entre le tronc vagal droit et son homologue gauche sur leur trajet intra-thoracique. Par ces rameaux, quelques filets afférents issus du vague abdominal gauche peuvent en effet parvenir au vague thoracique droit (ou inversement pour la déafférentation contralatérale). Cette suppression d'une grande partie des afférences vagales conduit néanmoins à d'importantes modifi- 
cations de 1'activité électromyographique (E.M.G.) postprandiale de l'antre gastrique (IAPLACE et DARCy, I978; DARCy et al., I979) : au cours des 6 h consécutives à un repas d'épreuve, le pourcentage d'activités électriques de contrôle (E.C.A.) auxquelles est associée une activité de réponse (E.R.A.) reste proche de go p. roo au liew de décroître régulièrement. Cette hyperactivité antrale peut être interprêtée comme la conséquence de la suppression du contrôle par rétroaction inhibitrice (réflexes entérogastriques d'origine duodénale) du fait de la déafférentation subtotale. Il nous a paru nécessaire d'évaluer directement les effets de cette intervention à l'égard de l'évacuation gastrique.

\section{Matériel et méthodes}

Trois porcs mâles castrés de race Large White, pesant respectivement 5055 et $5^{8} \mathrm{~kg}$ sont utilisés. Sous anesthésie générale à l'halothane, une canule en vinyle de diamètre intérieur/extérieur $22 / 30 \mathrm{~mm}$ est mise en place dans l'estomac à mi-hauteur de la grande courbure. Elle est extériorisée au niveau de la jigne blanche immédiatement en arrière de 1'appendice xyphoïde. L'inocuité de cette préparation à l'égard des activités électromyographiques gastriques a été vérifiée LAPLACE, I979a).

Ces porcs sont accoutumés à n'ingérer qu'un seul repas quotidien, distribué systématiquement le matin à $9 \mathrm{~h}$ oo. L'aliment utilisé est un aliment semi-synthétique composé de $82 \mathrm{p}$. Ioo de maïs, 3 p. Ioo de cellulose purifiée et I 5 p. Ioo d'un complément azoté, dont 1'évacuation par l'estomac est par ailleurs connue sur la base de mesures effectuées au niveau d'une fistule duodénale réentrante (CUBER et LAPLACE, I979). Dans le présent travail, l'évacuation gastrique de la matière sèche (détermination après $24 \mathrm{~h}$ à l'étuve à IO4 ${ }^{\circ} \mathrm{C}$ ) est fondée sur la collecte des quantités résiduelles présentes dans l'estomac à divers temps après l'ingestion du. repas d'épreuve, et le calcul par différence de la quantité émise dans l'intervalle de temps considéré.

Avant l'ingestion du repas d'épreuve, on procède à l'ouverture de la fistule gastrique. L'animal est abreuvé (500 ml) de façon à débarrasser l'estomac des ultimes résidus du repas de la veille. La vacuité gastrique étant ainsi assurée, et la canule refermée, le porc reçoit le repas d'épreuve ( $880 \mathrm{~g}$ de matière sèche). L'ouverture de la fistule n'intervient ensuite qu'une seule fois par épreuve à l'un des temps suivants : $30 \mathrm{mn}, \mathrm{I} \mathrm{h}, 2 \mathrm{~h}, 4 \mathrm{~h}$ ou $7 \mathrm{~h}$ après le repas. Les jours d'épreuve, le porc reçoit alors en remplacement une quantité d'aliment équivalant à celle dont il se trouve privé sur la base de la matière sèche moyenne des effluents gastriques.

La même méthodologie est appliquée après déafférentation vagale subtotale réalisée selon DARCY et al. (I979) c'est-à-dire par ablation du ganglion plexiforme gauche et vagotomie tronculaire droite au niveau du diaphragme. La déafférentation contralatérale n'a pas été mise en œuvre.

\section{Résultats et discussion}

Les quantités résiduelles moyennes de matière sèche, recueillies à chacun des temps de collecte par la fistule gastrique, sont après déafférentation vagale subtotale significativement plus importantes que chez le porc intact $(\mathrm{P}<0,05$ à 
$30 \mathrm{mn}$ et I h, $\mathrm{p}<\mathrm{o}, \mathrm{OOI}$ aux stades ultérieurs). Ceci signifie que 1'évacuation gastrique se trouve considérablement ralentie après déafférentation vagale subtotale (figure I).

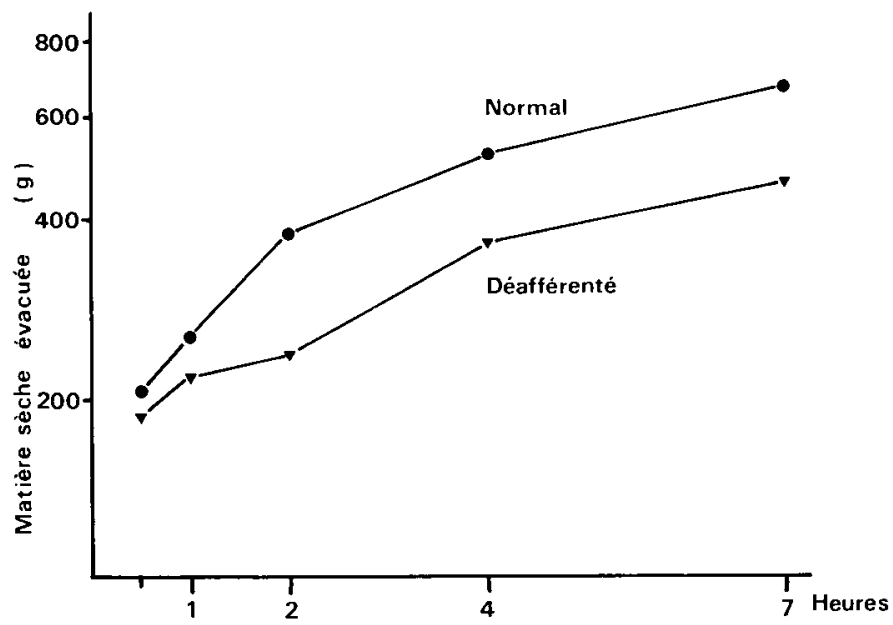

FIG. I. - Quantités moyennes de matière sèche évacuées par l'estomac chez le porc normal et chez le porc déafférenté (suppression chirurgicale sélective des voies vagales afférentes i.e. sensitives) $3^{\circ} \mathrm{mn}, \mathrm{I} h, 2 \mathrm{~h}, 4 \mathrm{~h}$ et $7 \mathrm{~h}$ après ingestion de $880 \mathrm{~g}$ (M.S.) d'un aliment à base d'amidon de mä̈s purifié.

Mean quantities of dry matter emptied by the stomach of a normal and a deafferented pig (selective surgical suppression of the vagal afferent fibers) at $30 \mathrm{mn}, \mathrm{I} h, 2 h, 4 h$ and $7 h$ after ingestion of $880 \mathrm{~g}(D . M$.) of a purified maize starch based diet.

Ce fait contraste singulièrement avec les images d'exacerbation des activités E.M.G. postprandiales de l'antre gastrique. Il reste plausible que la prolongation de la période postprandiale d'hyperactivité antrale relève d'un défaut de contrôle par voie réflexe à partir des récepteurs duodénaux (DARCy et al., I979). Mais la lenteur de l'évacuation gastrique suggère que les contractions antrales qu'impliquent les E.R.A. sont inefficaces, soit par défaut de coordination des activités antrales elles-mêmes, soit par perturbation de la coordination des activités antrales et duodénales juxta-pyloriques. Il faut aussi souligner que la déafférentation vagale subtotale préserve autant de voies motrices que la seule vagotomie tronculaire unilatérale réalisée au niveau du diaphragme. Or celle-ci n'entraîne aucune perturbation. La réduction importante đu volume des afférences vagales d'origine digestive s'avère donc réellement responsable à la fois des modifications de l'activité E.M.G. antrale et de celles de l'évacuation gastrique. Il n'en reste pas moins difficile de saisir par quel mécanisme ces effets sont produits.

MIoLAN et Roman (I978) ont montré que les afférences provenant de récepteurs gastriques peuvent induire, lors de distension de l'estomac, une augmentation de la fréquence de décharge des fibres vagales efférentes excitatrices; à l'inverse cette fréquence de décharge est réduite lors de distension duodénale. Ceci tend à montrer que les variations des décharges vagales efférentes correspondent à un phénomène de rétroaction c'est-à-dire à leur modulation par les afférences en provenance de récepteurs gastriques ou duodénaux.

Chez nos porcs, après déafférentation subtotale, la suppression des afférences d'origine gastrique implique l'absence d'hyperactivité vagale efférente. L'hyperac- 
tivité E.M.G. antrale postprandiale est pourtant présente; le caractère incomplet de la déafférentation ne peut être ici incriminé comme responsable, par le maintien d'un certain volume d'afférences gastriques, puisque la déafférentation totale (LAPLACE, I979b) entraîne également hyperactivité E.M.G. et stase gastrique. Par conséquent, l'existence chez le porc après déafférentation subtotale d'une hyperactivité E.M.G. antrale reposerait plutôt sur un mécanisme intrapariétal, que celui-ci soit actif même chez l'animal intact, ou seulement en substitution chez l'animal déafférenté. On conçoit aisément que la suppression des afférences d'origine duodénale, impliquant la perte des réflexes inhibiteurs duodéno-gastriques (i.e. la non réduction du volume de décharge efférente) contribue à la prolongation de l'hyperactivité antrale. Mais quel serait alors le mécanisme du défaut de coordination des activités motrices si telle était 1'origine du ralentissement de 1'évacuation gastrique?

Ce possible défaut de coordination des activités motrices pourrait éventuellement traduire un état d'hyperexcitation relative de la musculature antrale du fait de la mise en jeu du mécanisme excitateur intrapariétal et de la non inhibition des décharges vagales efférentes par défaut de rétroaction d'origine duodénale. On notera dans ce sens que l'excitation de la musculature antrale par stimulation électrique des branches vagales destinées à l'antre gastrique (SARNA et DANIEL, I975, I976) engendre, sous certaines conditions d'amplitude et de fréquence, une perturbation de l'activité électrique de contrôle antrale et l'apparition de contractions antrales et duodénales non coordonnées entre elles.

On fera également le parallèle entre cette apparence contradictoire des effets de la déafférentation vagale subtotale (hyperactivité E.M.G. antrale et évacuation gastrique ralentie) et la non moins surprenante contradiction relevée dans les effets de la gastrine. En effet, quoique la gastrine ait des effets excito moteurs à l'égard de l'antre gastrique (KWONG et al., I97I; WATERFALI, DUTHIF et BRown, I973) et qu'elle abaisse parallèlement la pression dans l'anneau pylorique (FISHER, LIPSHUTz et COHEN, I973), elle ralentit notablement 1'évacuation gastrique d'un repas d'épreuve (HUNT, I967) ou d'une solution saline (DozoIs et KELL, I, I97I). Aussi la gastrine, normalement libérée sous l'influence de réflexes nerveux vago-vagal ou local intramural, pourrait également être suspectée de jouer un certain rôle dans les effets de la déafférentation vagale.

\section{En conclusion}

La déafférentation vagale subtotale, quoique responsable d'une hyperactivité E.M.G. antrale, entraîne un retard considérable de l'évacuation gastrique. L'éventuelle responsabilité d'un défaut de coordination des activités antrales ou gastroduodénales devra être confirmée avant d'en rechercher les mécanismes nerveux et/ou humoraux. 


\section{Summary}

\section{Gastric emptying after subtotal vagal deafferentation in the pig}

The effects of the subtotal vagal deafferentation on gastric emptying of dry matter were studied in 3 pigs using a pertnanent gastric cannula. The selective surgical suppression of the vagal afferent fibers markedly delayed gastric emptying (fig. I). The apparent opposition between the postprandial antral hyperactivity, previously demonstrated, and the slow rate of gastric emptying is discussed in relation with the significance of the efferent vagal discharges in coordinating antral motility and with the role of the vagal afferences from stomach and duodenum in the modulation of these efferent discharges.

\section{Références bibliographiques}

CUBER J. C., IAPLACE J.-P., I979. Évacuation gastrique de la matière sèche d'un régime semipurifié à base d'amidon de maïs chez le Porc. Ann. Biol, anim. Bioch. Biophys., 19, 899-905.

Darcy B., Falempin M., Laflace J.-P., Roussfiat J.-P., I979. Importance de la voie vagale sensitive : recherche d'une technique de déafférentation sélective chez le porc et le mouton. Ann. Biol. anim. Bioch. Biophys., 19, 881-889.

DozoIs R. R., KEI I Y K. A., I97I. Effect of a gastrin pentapeptide on canine gastric emptying of liquids. Amer. J. Physiol., 221, I I3-I 7 .

FISHER R. S., LIPSHCTZ W., COHEN S., I973. The hormonal regulation of pyloric sphincter function. J. Clin. Invest., 52, I $289-1296$.

HUNT J. N., I967. Fffect of gastrin II on gastric emptying and secretion during a test meal. Brit. Med. J., 4, 386-387.

Kwong N. K., Brown B. H., Whittaker G. E., Dethie H. L., 1971. Response of the electrical activity, motor activity and acid secretion of the human stomach to pentagastrin and histamine stimulation. Scand. J. Gastroent., 6, I 45-I53.

LAPLACE J.-P., I979a. Stomach and small intestine motility in the Pig. Electromyography in nutritional studies in Seminar on Digestion and Absorption in cannulated pigs, Shinfield-U.K., (sous presse).

IAAPIACE J.-P., I979 b. Surgical deprivation of vagal afferences from gastro-intestinal tract of the pig. Clinical and E.M.G. studies. $7^{\text {th }}$ Int. Symp. on Gostrointestinal Motility, Iowa CityU.S.A. (sous presse).

LAPLACE J.-P., DARCY B., I978. Gastric motility in the Pig after surgical interruption of vagal afferent pathways, K.A. 44 in 5 th Int. Pig. Vet. Soc. Congress, Zagreb.

MIOLAN J.-P., ROMAN C., I978. Discharge of efferent vagal fibers supplying gastric antrum: indirect study by nerve suture technique. Am. J. Physiol., 235, E 366-E 373 .

SARNA S. K., DANIEL E. E., I975. Vagal control of gastric electrical control activity and motility. Gastroenterology, 68, 30I-308.

SARNA S. K., DANiEL E. E., I976. Neuronal control of motility in the pyloric region in dog. Gastroenterology, 70, 933 (Abstr.).

WATERFALI, W. E., DUTHIE, H. I. BRown B. H., I973. The electrical and notor actions of gastro intestinal hormones on the duodenum in man. Gut, 14, 68g-696. 